\title{
Combatting infectious diseases; nanotechnology as a platform for rational vaccine design ${ }^{\text {tr }}$
}

\author{
Elly van Riet ${ }^{\mathrm{a}, 1}$, Akira Ainai ${ }^{\mathrm{a}, \mathrm{b}}$, Tadaki Suzuki ${ }^{\mathrm{b}}$, Gideon Kersten ${ }^{\mathrm{c}, \mathrm{d}}$, Hideki Hasegawa ${ }^{\mathrm{b}, *}$ \\ a Influenza Virus Research Centre, National Institute of Infectious Diseases, Gakuen 4-7-1, Musashimurayama-shi, Tokyo 208-0011, Japan \\ b Department of Pathology, National Institute of Infectious Diseases, Toyama 1-23-1, Shinjuku-ku, Tokyo 162-8640, Japan \\ ${ }^{c}$ Institute for Translational Vaccinology (Intravacc), P.O. Box 450, 3720 AL Bilthoven, The Netherlands \\ d Division of Drug Delivery Technology, Leiden Academic Centre for Drug Research, Leiden University, PO Box 9502, 2300 RA Leiden, The Netherlands
}

\section{A R T I C L E I N F O}

Available online 23 May 2014

\section{Keywords:}

Nanoparticle

Pathogen

Adjuvant

Administration route

Delivery

Targeting

Innate immunity

Adaptive immunity

\begin{abstract}
A B S T R A C T
Currently, several successful vaccines are available. However, for pathogens with a highly variable genetic composition, and for which serum IgG antibodies are not a useful correlate of protection, effective vaccines are yet to be developed. This is due to a lack of both the understanding of the immunological pathways leading to longterm protection and the ability to translate the available knowledge into a suitable vaccine formulation. Regarding the latter, nanoparticles can be an attractive platform for vaccine development, as they offer multiple options for improving safety and efficacy. For example, side effects might be decreased upon encapsulation of the adjuvant and the concomitant delivery of antigen and adjuvant is a very promising tool for increasing efficacy. In addition to the many promises, the use of nanoparticles as vaccine carriers should be implemented with caution: the more sophisticated a particle, the more parameters need to be controlled during production and storage. (c) 2014 Published by Elsevier B.V.
\end{abstract}

\section{Contents}

1. Introduction . . . . . . . . . . . . . . . . . . . . . . . . . . . . . . . . . . . . . . . . . . . . . . . . 29

2. Immunological considerations in vaccine design . . . . . . . . . . . . . . . . . . . . . . . . . . . . . . . . . . . . . . . . 29

3. Nanoparticle vaccine delivery . . . . . . . . . . . . . . . . . . . . . . . . . . . . . . . . . . . . . . . . . . . 29

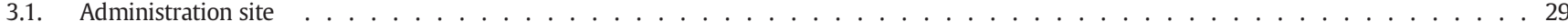

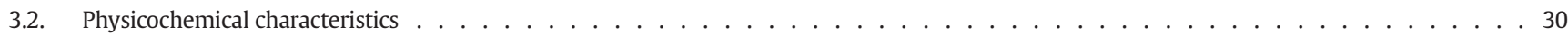

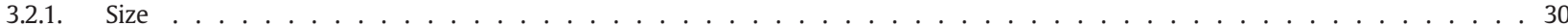

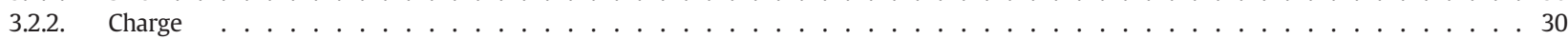

3.2.3. Hydrophobicity . . . . . . . . . . . . . . . . . . . . . . . . . . . . . . . . . . . . 30

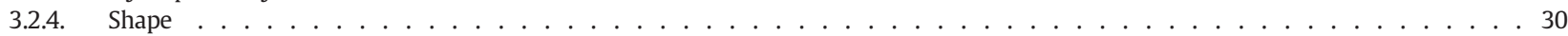

3.2.5. Surface organization . . . . . . . . . . . . . . . . . . . . . . . . . . . . . . . . . . . 30

3.3. Immunomodulation . . . . . . . . . . . . . . . . . . . . . . . . . . . . . . . . . . . . . . . . 31

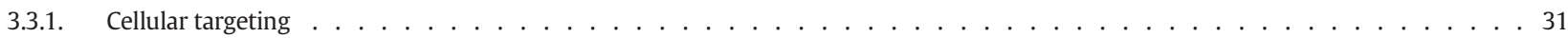

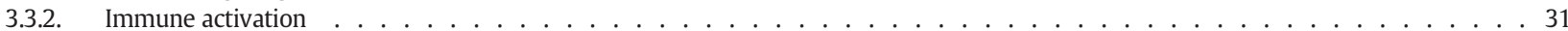

3.4. Control of antigen release . . . . . . . . . . . . . . . . . . . . . . . . . . . . . . . . . . . . . . . . 32

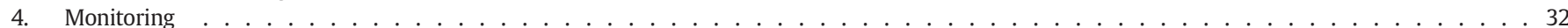

5. Safety . . . . . . . . . . . . . . . . . . . . . . . . . . . . . . . . . . . . . . . . . 32

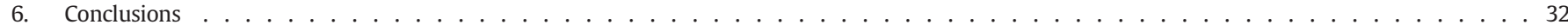

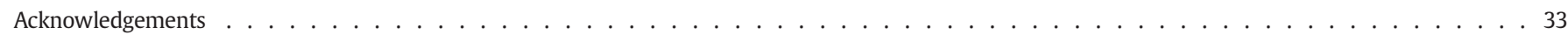

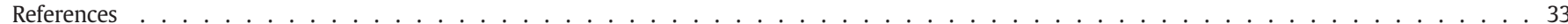

This review is part of the Advanced Drug Delivery Reviews theme issue on "Nano-pathophysiology: a novel integrated approach to disease through application of nanotechnology".

* Corresponding author.

E-mail addresses: elly.van.riet@intravacc.nl (E. van Riet), hasegawa@nih.go.jp (H. Hasegawa).

1 Present address: Institute for Translational Vaccinology (Intravacc), P.O. Box 450, 3720 AL Bilthoven, The Netherlands. 


\section{Introduction}

Prophylactic vaccination arguably is the most successful and costeffective medical intervention available to mankind. Intensive childhood vaccination programs have drastically reduced morbidity and mortality due to several types of infectious diseases including diphtheria, pertussis, tetanus, polio, measles, mumps, rubella and smallpox, as illustrated by an over $90 \%$ decrease in cases and over $99 \%$ decrease in deaths in the USA since the introduction of the respective vaccines $[1,2]$. However, for many other pathogens an effective vaccine is not available yet. The most successful vaccines currently in use were generated empirically, which was effective because the pathogens they protect from were not able to escape immune responses. In contrast, diseases for which efficient vaccines are not currently available, are mostly caused by pathogens mastering either immune escape or immune evasion.

For example, RNA viruses like human immunodeficiency virus (HIV) and influenza virus have the capacity to quickly change their surface antigens due to sequence variability and glycosylation. Thus, in response to for example human neutralizing antibodies, new viruses will emerge that cannot be recognized by these antibodies [3-5]. Alternatively, large DNA viruses such as herpesviruses and poxviruses can evade the host immune responses via immunomodulatory pathways, such as suppression of antigen presentation, suppression of co-stimulation, production of immunosuppressive cytokines or obstruction of pathways of proinflammatory cytokines [4]. These evasion strategies are also used by other pathogens such as the bacterium Mycobacterium tuberculosis [6] and parasites like for example malaria [7].

Since many pathogens master the art of outwitting host immune responses raised against them, new vaccines will have to be developed that raise strong and broad immune responses that are difficult for the pathogen to counteract. Importantly, the ability to take vaccinology one step further is not only dependent on increasing basic immunological knowledge but also on the development of advanced materials and techniques [8] to implement the knowledge into products that are safe, efficient, stable and cost-effective. For this, nanotechnology is a very useful and promising field. Athough the concept is not new, with liposomes and microparticles for use as vaccine adjuvants already reported in the 1970s [9,10], and immune stimulating complexes (ISCOMs) in the early 1980s [11], currently knowledge is increasing on how nanotechnology can be used to rationally optimize vaccine formulations $[12,13]$.

Here we have attempted to give an overview of the main immunological aspects in the design of prophylactic vaccines and how these immunological challenges can be addressed by implentation of nanotechnology in vaccine design to prevent infectious diseases.

\section{Immunological considerations in vaccine design}

In essence, what a vaccine is supposed to do, is activating the immune system to raise a protective response against the antigen(s) present in the formulation that will ensure protection upon a subsequent encounter of the host with pathogens bearing (parts of) those antigens, any time after the vaccination. To establish this, the innate immune system needs to be activated and in addition ideally an antigen containing epitopes that can interact with B-cell receptor needs to be included as well as antigens that can be presented on Major Histocompatibility Complex (MHC)II and MHCI molecules, for activating helper (CD4) and killer (CD8) T cells, respectively. Such a diverse immune response will decrease the likelyhood of immune escape and also maximize the number of responsive people [14]. However, it should be taken into account that B-cell epitopes must be formulated in such a way that sufficient eptitopes remain intact up to the point where they interact with B-cells. In contrast, T-cell epitopes are formed in vivo by antigen processing, and thus efficient uptake by antigen presenting cells, preferably dendritic cells (DC), should be optimized [15].
Searching for suitable antigens includes selection for several characteristics, including immunogenicity [16] and conserved presence of this antigen in as many strain variants of the pathogen as possible since these antigens are more likely to be crucial for the fitness of the pathogen and consequently it might be more difficult for the pathogen to evade the immune system as it might not be able to survive when mutations occur in these genes. In addition, for $T$ cell epitopes, the majority of the common human Human Leucocyte antigen (HLA) types should be covered [17].

Importantly, also care should be taken that the selected antigen(s) do not contain an epitope that might reduce the intended immune responses $[18,19]$.

When a useful set of antigens is selected, they should be formulated in such a way that a robust immune response is induced. For this, additional components can be added to the formulation to enhance the immune response to an antigen. These are called adjuvants, derived from the latin 'adiuvare', meaning 'to aid'. However, in the case of nanoparticle-based vaccines, several components can be added that might not traditionally be called 'adjuvants', as they more indirectly optimize the immune response. For example, targeting the particles to certain cell types might not activate those cells more than the antigen alone, but it could be crucial for the induction of the appropriate type of immune response. Since several molecules can be combined in a nanoparticle, it could be stated that the combined characteristics of the components of the particle, except the antigen itself, together represent the overall adjuvant function [20].

Equally important to the adjuvanticity, an adjuvant should also be safe. A component activating the innate immune system should be carefully selected and formulated. Since that is usually the most immunogenic part of a vaccine, it also harbours the most risks on side effects and in case of preventive vaccination, even minor effects might prevent acceptation of the vaccine.

\section{Nanoparticle vaccine delivery}

Delivery of nanoparticulate vaccines can be addressed to 3 different levels. First, the route of administration; in case of prophylactic vaccines to protect from infectious diseases, delivery to mucosal tissues in the nose or mouth and different areas of the skin are the most practical. Second, by adding ligands that bind to receptors only present on a particular cell type, the vaccine can be specifically delivered to certain cells. Finally, by targeting a specific type of receptor on the cells, it can be influenced to which intracellular compartment the antigen will be directed and thus where and how it will be processed and which type of immune response will be induced. These different levels of delivery are influenced by several vaccine characteristics as described below.

\subsection{Administration site}

Currently, most vaccines are administered intramuscularly; however, other administration routes are widely explored. For example, for induction of local protection, local vaccination has been proven superior in many cases. In addition, alternative administration routes have some practical advantages, such as allowing easier administration and reduction of needle waste [15]. Alternative administration routes require specific characteristics of the nanoparticle carrier. For example, antigens are not often efficiently crossing the nasal mucosa upon nasal vaccination. This can be improved by the use of nanoparticulate antigen carriers that are composed of, or coated with, mucoadhesive polymers, or by actively targeting cells that can transport the particles across this barrier [21].

In the case of (epi)dermal vaccination, several methods have been developed to enable vaccine formulations to cross the natural barrier of the skin, the stratum corneum, including microneedles, skin disruption methods and jet injectors [22]. And for oral vaccination the vaccine 
formulation should be able to resist the low $\mathrm{pH}$ and enzymatic destruction in the digestive tract [23].

Since the route of administration greatly influences the localization in the body where the vaccine is delivered, it is important to realize that this will also affect immune responses. Protection by current available succesful vaccines in humans relies mostly on strong systemic immunoglobulin (Ig)G responses and thus vaccine design has mostly focussed on induction of IgG, sometimes in combination with Thelper (Th)1 type CD4 T helper cells and CD8 cytotoxic T-cells. However, one should keep in mind that this knowledge is mainly based on experiments with cells isolated from the blood and upon intramuscular vaccination. Importantly, those results cannot be extrapolated to draw conclusions on responses in other organs and tissues. For example, the cytokine TGF- $\beta$ is usually associated with tolerance, but TGF- $\beta 1$ is also very important for induction of IgA class switching, and might thus be very interesting for induction of mucosal protection [24-26]. Although it is often posed that the type of immune response that is protective is dependent on the type of pathogen, it is becoming increasingly clear that the optimal immune response is dictated by the pathogen in combination with the tissue where the response is induced [26].

\subsection{Physicochemical characteristics}

Live attenuated pathogens, or antigens formulated in a particle, are much more effectively taken up by antigen presenting cells than antigens in subunit vaccines, because particles have large surfaces with characteristics that increase the interaction with antigen presenting cells. Many types of nanoparticles can be used, such as liposomes, ISCOMs, Virus Like Particles (VLPs), polymeric nanoparticles and nondegradable nanoparticles [27]. The strength and type of interaction are influenced by several physicochemical characteristics of the particles such as the size, the geometry and surface characteristics such as zeta potential and the degree of hydrophilicity or hydrophobicity [28].

\subsubsection{Size}

When a vaccine formulation enters the body, transport is highly dependent on the size. Upon intramuscular administration, the optimal size for entering the lymphatic system is about $40 \mathrm{~nm}$, with a range of 20-200 nm. Particles larger than 200-500 nm are mostly carried into the lymphatic system by specialized cells, such as DC. Direct drainage of small molecules via the lymphatic system is fast and happens within a few hours of administration, whereas it takes about $24 \mathrm{~h}$ for a molecule to reach the lymph nodes when it is taken up by DC and subsequently together traffic into and along the lymphatic system [28]. These forms of transport as well as the related kinetics will also influence the types of cells that are activated.

In addition, size will influence the extent of cytotoxicity of nanoparticles. In non-phagocytic cells, smaller size was associated with an increased cytotoxicity [29]. However, for phagocytes different results were found, with particles in the range of $20-200 \mathrm{~nm}$ being less toxic than microparticles [29].

\subsubsection{Charge}

Since cell membranes are anionic in nature, it could be expected that cationic particles are more efficiently taken up, and this is also mostly found [27], although it has also been reported to be different for phagocytes [29]. It is important to consider that charged particles also induce cytotoxicity, with cationic and anionic particles generally causing membrane damage and intracellular damage, respectively [29]. In addition, surface charge was found to influence intracellular localization with negatively and neutrally charged nanoparticles co-localizing with lysosomes, whereas positively charged nanoparticles could escape from lysosomes after being internalized [30]. However, it should be kept in mind that the zeta potential of nanoparticles also depends on environmental factors as their charge can be shielded by counter ions [30].

\subsubsection{Hydrophobicity}

The nanoparticle materials used, will also affect their hydrophobicity. It has been reported that the degree of phagocytosis of bacteria was correlated with their hydrophobicity and hydrophobic polymers were shown to cause more inflammation than hydrophilic polymers [31]. Interestingly, also many pathogen-derived ligands that activate receptors of the innate immune system, such as lipopolysaccharide (LPS), bacterial fimbriae and the fusion protein of respiratory syncytial virus contain hydrophobic parts that are exposed upon damage. In all cases tested, the hydrophobic parts of the molecules co-purified with the immunostimulatory portions. Moreover, many adjuvants contain large hydrophobic sites, including all recently licensed adjuvants: MPL-plusalum and the oil-in-water emulsions MF59 and AS03 [31,32].

\subsubsection{Shape}

Another characteristic influencing cellular uptake can be the shape of a nanoparticle. Phagocytosis of particles requires a certain actin structure to be created that allows the membrane to move over the particle to internalize it. The complexity of the actin structure that must be created depends on the curvature at the initial point of contact, and therefore worm-like particles or particles with an elliptical shape were found to only be internalized when the ends of the particle, with a high curvature, like spherical shapes, were attaching to the cell [33,34]. However, in other studies it was found that sphereocylindrical or rod-shaped nanoparticles were endocytosed more efficiently [35,36]. The reason for that difference remains to be investigated. Possibly the spacing and organization of surface molecules interferes with cellular attachment and uptake. In addition, how shape might affect receptor-mediated uptake still needs to be investigated.

\subsubsection{Surface organization}

Surfaces of viruses and bacterial structures such as flagella are also polyvalent and in addition highly organized, as they usually contain only a few proteins, each present not only in large amounts but also in an ordered manner [28]. This antigen organization and repetitiveness form a typical characteristic of pathogens and trigger the immune system.

For B-cell activation it was determined that 15-20 molecules with a space of 5-10 $\mathrm{nm}$ in between are very potent inducers of antibody responses [37]. When nanoparticles were created with 60 peptide epitopes displayed per particle, with spaces of 5-10 nm, these were able to optimally induce antibody responses [28]. Interestingly, components of the humoral innate immune system generally also have a multimeric structure, possibly explaining the pronounced effect of antigen organization and repetitivenes on induction of antibody responses [38].

In addition to repetitiveness of the antigen, also the accesability of different epitopes is a prerequisite for induction of a robust immune response, as illustrated by the spacing needed in between the epitopes and in addition it is dependent on the conformation of the antigen. This was nicely demonstrated for an influenza vaccine based on ferritin-hemagglutinin (HA) fusion proteins that could self-assemble into nanoparticles in which the HA could form the physiological relevant symmetric homotrimeric viral spike. When these nanoparticles were used to immunize mice, it was shown that slightly higher antibody levels were induced compared to trivalent inactivated virus, that could be increased even further by the addition of an oil-in-water adjuvant (ribi or MF59). Interestingly, the nanoparticle formulation induced antibodies that were not only neutralizing the vaccine strain, but also relating strains, due to antibodies recognizing conserved regions in the HA stem or head [39]. Similarly, cross-reactivity was found to be increased by using nanoparticles made of the coat protein of papaya mosaic virus. The expression of this protein in bacteria led to self-assembly and the formation of highly organized VLPs. This surface rearrangement also led to formation of antibodies reactive against a highly conserved region of the HA antigen [40]. 
This effect of natural conformation cannot only be applied to antigens, but has also been found for targeting ligands; ligands of the lectin receptor DC-specific ICAM-3-grabbing nonintegrin (DC-SIGN) are recognized with higher affinity by their tetrameric receptor when exposed in a multivalent form [41].

\subsection{Immunomodulation}

As a result of differences in the physicochemical properties of the particles, the quality of the immune response may be affected. For example, it was reported that amino-functionalized, but not carboxyl-, or non-functionalized, polystyrene nanoparticles of $\sim 100 \mathrm{~nm}$ induced lysosomal rupture followed by NLRP3 inflammasome activation [42]. In another study this lysosomal escape was found to be related to the shape of the particles. In this study it was investigated how gold nanoparticles, coated with West Nile envelope protein, of different size and shape affected immune responses. Larger spherical particles (40 nm) were found to mostly remain in the lysosome and induce TNF- $\alpha$ whereas particles of $20 \mathrm{~nm}$ in shape as well as rods could enter the cytosol. However, only in the case of the rods this lysosomal escape resulted in cytotoxicity and expression of the cytokines IL-1 $\beta$ and IL-18, implicating a role for NLRP3 inflammasome signaling [36].

\subsubsection{Cellular targeting}

To initiate an antigen specific $\mathrm{T}$ cell response, the antigens generally need to be processed by antigen presenting cells, and subsequently presented to CD4 and/or CD8 T cells. Many cells including macrophages, some endothelial cells and also B cells can function as antigen presenting cells. The most professional and most intensively studied antigen presenting cell is the DC; however, many types of DC subsets have been discovered, which differ in their surface receptors, location and funtions [43-46]. Thus, depending on the type of immune response that is needed to induce a robust immune response, a different antigen presenting cell could be targeted by adding receptor-interacting surface molecules.

All dendritic cells express a variety of surface expressed pattern recognition receptors that can be used to target these cells, such as some of the Toll-like receptors (TLRs) [44,47], and C-type lectin receptors. Several of the ligands binding to these innate receptors are currently explored for their possible application in vaccines, such as TLR2 and TLR4 agonists, glycans activating C-type lectin receptors [48] or combinations of both [41]. In the latter case, it was found that CD8 T cell proliferation was greatly enhanced by the combination of DC-SIGN targeting with TLR4, but not TLR9, activation using lipopolysaccharide (LPS) and unmethylated CpG dinucleotides, respectively [41]. However, when TLR2 agonist PAM was used as a targeting moiety for virus-like particles, this hardly increased dendritic cell uptake but it did induce DC maturation at very low doses [49], illustrating that it is difficult to distinguish targeting ligands from immune activating ligands as one molecule might be able to do both, depending on the circumstances.

Alternatively, antibodies that bind surface molecules on DC can be used to trigger the innate immune system, such as $\alpha$ DEC205 [50] and $\alpha \mathrm{CD} 11 \mathrm{c}[51]$.

Regarding targeting of DC via DC-SIGN it was found that poly(lacticco-glycolic acid) (PLGA) nanoparticles coated with polyethylene glycol and DC-SIGN binding antibodies increased antigen uptake and internalization compared to targeting using coating of the nanoparticles with carbohydrates that can act as DC-SIGN ligands. Since the carbohydrates and the antibodies bound to the same domain of DC-SIGN and in addition the loading density of the DC-SIGN ligand was higher compared to that of the $\alpha$ DC-SIGN antibody, this increased uptake of antibody coated nanoparticles is most likely due to a higher affinity of the antibodies for the binding region [52]. Nanoparticles combining the presence of antibodies recognizing DC-SIGN on their surface and encapsulating a TLR ligand strongly increased immune responses as these nanoparticles were 100 -fold more potent in inducing the maturation markers CD80 and CD83 than non-targeted nanoparticles [53]. When $\alpha$ DEC205 was used as a targeting ligand it was found that targeted TLR ligands were required in a 100 fold lower dose compared to the soluble ligands to induce similarly strong cytotoxic T cell responses. Not surprisingly, the doses of soluble TLR ligands used to induce potent cytotoxic T cell responses were extremely toxic, whereas the targeted delivery of the TLR ligands not only requires lower amounts, but in addition TLR ligands delivered to cells induce less systemic toxicity. The strong responses with respect to DC maturation and cytotoxic $\mathrm{T}$ cell responses can be very useful in vaccine development. However, especially for use in prophylactic as compared to therapeutic vaccines, these responses will require further investigation in order to understand the mechanisms and response limits with respect to possible side effects.

Interestingly, it was found that targeting of antigen and adjuvant to the same DC population alone is not enough. Another important characteristic involved was timing. When antigen reached the DC before the adjuvant, the DCs gained a non-activated phenotype and interfered with the intended immune polarization [54].

The reverse, first adjuvant and next antigen seemed to induce similar or even better responses than delivery at the same time. For nanoparticle vaccines this implicates firstly that nanoparticles are a very interesting delivery vehicle indeed, as the possible combined delivery of antigen and adjuvant to the same cell is one of the features of nanoparticle vaccines. Second, this also implicates caution, as no antigen should be released from the particles, either during storage or upon vaccination before it is delivered to a (dendritic) cell, as the resulting soluble antigen would likely decrease the efficacy of the vaccine.

\subsubsection{Immune activation}

As discussed above, targeting ligands influence the type of cell that is targeted, but also the degree and mode of response of this cell. Targeting a certain C-type lectin receptor will activate other immune mechanisms than targeting a different lectin receptor or a Toll-like receptor. In addition, after internalization also intracellular receptors can be activated such as the internal TLRs (TLR3, 7, 8 and 9) [55], RigI helicases [56] and Nod-like receptors. Some members of the latter family are involved in the formation of so-called inflammasomes [57].

Vaccine delivery systems can not only target certain cell types, but they can also influence to which intracellular compartments the antigen will be transported and where it will be processed, thereby influencing (1) whether or not the immune system is responding [58], (2) which types of cells are responding $[59,60]$ and (3) the type of immune response that is induced $[61,62]$. For example, it was found that nanoparticles composed of PLGA, PLGA-N-trimethyl chitosan (TMC) and TMCtripolyphosphate (TPP), all containing ovalbumin as an antigen, were inducing different responses. These particles had a similar size (250$500 \mathrm{~nm}$ ), but different surface charge and antigen release kinetics. Upon intranasal vaccination in mice, all formulations induced CD4 T cell proliferation, especially PLGA and PLGA-TMC. However, the PLGA containing particles enhanced CD4 T cell mediated immune tolerance and did not induce humoral responses after intranasal administration, although they did after intramuscular treatment. In contrast, TMC-TPP did not induce tolerance, but strongly induced humoral responses [63].

Regarding the activation of CD4 compared to CD8 T cells, it was reported that if an antigen was taken up by the mannose receptor, it was transported to early endosomes and resulted in presentation exclusively on $\mathrm{MHCI}$, whereas transport to lysosomes, for example via the scavenger receptor or pinocytosis resulted exclusively in presentation by MHCII. Thus, this way either CD8 or CD4 T cells could be exclusively activated [59]. Interestingly, this specific targeting of CD4 or CD8 cells was also shown to be induced by certain particles: liposomes consisting of dioleoyl-phosphatidylcholine and dioleoyl-phosphatidylserine (DOPC/DOPS), resulted in delivery of the encapsulated antigen to late endosomal compartments, inducing MHCII presentation, whereas dioleoyl-phosphatidylethanolamine plus cholesteryl hemisuccinate 
(DOPE/CHEMS) liposomes delivered the antigen to early endosomal compartments and resulted in presentation on both $\mathrm{MHCI}$ and II [60]. For induction of CD8 T cell responses, cross-presentation is enabled upon cytosolic delivery of the antigen. For vaccine delivery, an efficient way to facilitate this cytosolic delivery might be the use of fusogenic vesicles, that, through fusogenic proteins or liposomes induce membrane fusion and subsequent direct cytosolic delivery of the vaccine to the cytosol $[64,65]$.

In case several molecules will be combined in a single nanoparticle, it will need thorough investigation how the concomitant activation of several pathways will influence the final immune response. Certain combinations of adjuvants have been reported to induce synergy [66]; however, it should be noted that the combination that induces the highest possible production of certain cytokines is not necessarily the best combination for induction of a broad, long lasting immune response without side effects.

\subsection{Control of antigen release}

The presence of antigen over a prolonged time allows for repeated interaction of the antigen with the immune system, leading to improved immune responses. Therefore the depot function provided by classical adjuvants like some alum formulations has long been recognized to contribute to their adjuvanticity.

However, during natural infections antigen are increasingly available to the immune system as usually only a few pathogens enter the body and start proliferating exponentially. These kinetics, that are representative of (highly) virulent pathogens might be a signal for the immune system to take it seriously and induce a strong immune response. This was nicely illustrated by a study showing that exponentially increasing antigen doses induced stronger CD8 T cell responses than the same amount of antigen added as a single dose in incomplete Freund's adjuvant or repeated injections of uniform doses, the latter inducing the weakest response [67]. In the same study it was shown that an initial high exposure followed by constant low rate exposure was less efficient than only a constant low rate exposure. Indeed, when a pathogen enters the host with great numbers and thereafter is quickly disappearing it might not represent a great danger. Exponentially increasing doses were not tested for B-cell responses; however, persistent presence of the antigen, mimicked by daily administration, induced higher antibody levels than weekly administration.

For anticancer treatment repeated injections in a short time frame might be feasible; however, in the case of prophylactic vaccines, that is not an option. Nonetheless, it is interesting to speculate about the possibility of entrapping increasing numbers of nanoparticles in increasingly stable microparticles. Administration of a mixture of these particles would provide an increasing release of antigen. Such a so-called multivalent single injection vaccine had been in development, based on PLGA microspheres containing different layers with antigen to produce a pulsatile release profile. In the near future, the expected cost increase accompanying these sophisticated techniques will likely be too big an economic hurdle for prophylactic vaccines, but it might get a followup in the future $[68,69]$.

\section{Monitoring}

For efficient monitoring of the effect of vaccination and to be able to compare different vaccine formulations, firstly the term 'protection' should be clearly defined. 'Protection in mucosal surfaces' will raise other correlates of protection than 'protection from symptomatic infection'. In addition, the definition of a correlate of protection depends on the pathogen, and the host (genetic background, sex, age) [70]. The 'ideal protective immune response' is currently not fully understood for most pathogens and finding usefull correlates of protection will remain one of the big challenges in vaccine design.
Further, it is crucial to monitor which immunological responses are induced by changes in vaccine design, both protective responses as well as unexpected side effects. Although general knowledge about the effect of physicochemical particle characteristics, adjuvant use and targeting is currently expanding, relatively little is known about how several characteristics of nanoparticles will influence one another. For monitoring the effects of slight changes in vaccine design in experimental settings, nanoparticles are very useful. By making use of the imaging options of nanoparticles itself, or by adding an imaging agent to the formulation, the in vivo biodistribution, including residence time, kinetics and which specific tissues and cells are targeted can be monitored in vivo $[71,72]$. The next step will be to relate these characteristics to the immunological outcome of the vaccination, thereby contributing to changing vaccinology into a field based on ratio instead of trial and error.

\section{Safety}

As discussed above, nanoparticle characteristics can be tailor-made, and their effects can be monitored to further improve the use of the nanoparticles in a rational way. However, the seemingly endless possibilities for application also imply caution: nanoparticles can be extremely complex, with all constituents having a specific function and in addition all characteristics possibly influence one another. This implies that the production of nanoparticles should be standardized, since for example a different spacing or symmetry of the surface molecules could lead to different targeting and thus possibly toxic effects instead of the effect that was intended to be induced. This was for example illustrated by the use of nab-paclitaxel, a nanoparticle drug that had proven safe. However, after the patent protection ended it was being copied by other companies than the innovator and those products showed to have very different characteristics, varying from too high endotoxin and residual solvent levels, inter-batch variations in particle size to poor stability at $40^{\circ} \mathrm{C}$, resulting in large aggregates [73]. To complicate it even further, characterization of nanoparticles often interferes with in vitro methods to evaluate their physical and immunological characteristics. For example, nanoparticles often absorb light or can have catalytic properties and are thus able to influence assays that rely on enzymatic reactions, as illustrated by the example of the endotoxin test, where polymer particles raised false positive results [74].

Therefore, for clinical use and production on a larger scale, the possible mechanisms of cytotoxicity related to the type of nanoparticle should be thoroughly investigated [75]. In addition, from the start of the development one should try and avoid steps that might play a role in safety issues. For example, it would be best not to use toxic solvents at any step in the process, and in addition the whole process should be extremely well controlled to ensure that no subtle changes will be introduced along the production line that might lead to changes in activity of the product. This is especially crucial in the case of the use of nanotechnology in vaccine design, since not only are vaccines usually administered to a large number of people, the people who receive prophylactic vaccines to protect them from infectious diseases are healthy and for many vaccines the majority are young children. Thus, in contrast to therapeutic vaccines for severely ill people, vaccines with even the slightest risk or side effect will not be accepted. However, regarding the latter, the human body does need a signal that indicates that there is a task to be completed, and this has so far proven impossible without the induction of any damage or 'danger'; thus, acceptance of a minor inconvenience (such as slight increase in temperature) should be explained to be necessary for effective vaccination and thus not a side effect, but an essential part of the effectiveness.

\section{Conclusions}

The use of nanoparticles as a platform for vaccine design holds many opportunities, resulting an increased interest by scientists, illustrated by 
an enormous increase in published articles: when searching in pubmed for the combination of 'nanoparticle' and 'vaccine' in the title or abstract, the first result is found in 1995, with 4 hits until 2000, 80 hits in the first decade of this century and 162 hits in 4 years starting from January 2010-an exponential increase. However, as discussed above, the use of nanoparticles in vaccines also implies caution as the more complicated the particle, the more difficult to control production and stability during storage and the more complex the immune pathways that are induced. In the case of prophylactic vaccination safety is of utmost importance; thus, implementation of nanoparticles in this field will require some hurdles to be taken. This is also reflected in their use for human applications. Although two prophylactic vaccines that use a nanosize carrier have been licenced, both based on VLPs and directed against hepatitis $B$ virus and human papillomavirus, the number of clinical trials with nanoparticles as registered at www.clinicaltrials.gov, a service of the U.S. National Institutes of Health, gives 163 reports (last accessed April 10th 2014). However, 125 are related to cancer, and of the 38 left, most are related to autoimmune diseases like diabetes. For prohylactic vaccines against infectious diseases only one vaccine can be found. Moreover, a closer look reveils that for this vaccine against respiratory syncytical virus alum is added to the nanoparticle formulation, resulting in large micron, instead of nano, sized particles [76,77]. However, there surely is hope for the future: in the veterinarian field advances are made [78], and combined with continuously increasing fundamental knowledge in both the immunological and technical field and knowledge generated in clinical trials using nanoparticles to treat cancer or autoimmune diseases, the nanoparticle platform will become more mature, with more successes to be expected.

\section{Acknowledgements}

E. van Riet is a recipient of a Postdoctoral Fellowship for Foreign Researchers of the Japan Society for the Promotion of Science. This study was further supported by grants from the Ministry of Health, Labour and Welfare, "H19-Trans-Ippan-002" and "H23-Shinkou-Ippan-015". Also, we would like to acknowledge all scientists contributing to this field of work, but whose work is not cited in this review.

\section{References}

[1] R. Rappuoli, H.I. Miller, S. Falkow, Medicine. The intangible value of vaccination, Science 297 (2002) 937-939.

[2] S.W. Roush, T.V. Murphy, Historical comparisons of morbidity and mortality for vaccine-preventable diseases in the United States, JAMA 298 (2007) 2155-2163.

[3] J.P. Julien, P.S. Lee, I.A. Wilson, Structural insights into key sites of vulnerability on HIV-1 Env and influenza HA, Immunol. Rev. 250 (2012) 180-198.

[4] R. Arens, Rational design of vaccines: learning from immune evasion mechanisms of persistent viruses and tumors, Adv. Immunol. 114 (2012) 217-243.

[5] C.D. O'Donnell, L. Vogel, A. Wright, S.R. Das, J. Wrammert, G.M. Li, M. McCausland, N.Y. Zheng, J.W. Yewdell, R. Ahmed, P.C. Wilson, K. Subbarao, Antibody pressure by a human monoclonal antibody targeting the 2009 pandemic H1N1 virus hemagglutinin drives the emergence of a virus with increased virulence in mice, mBio 3 (2012).

[6] P.S. Redford, P.J. Murray, A. O'Garra, The role of IL-10 in immune regulation during M. tuberculosis infection, Mucosal Immunol. 4 (2011) 261-270.

[7] C. Coban, K.J. Ishii, T. Horii, S. Akira, Manipulation of host innate immune responses by the malaria parasite, Trends Microbiol. 15 (2007) 271-278.

[8] M.A. Swartz, S. Hirosue, J.A. Hubbell, Engineering approaches to immunotherapy Sci. Transl. Med. 4 (148) (2012) 148rv9.

[9] A.G. Allison, G. Gregoriadis, Liposomes as immunological adjuvants, Nature 252 (1974) 252.

[10] G. Birrenbach, P.P. Speiser, Polymerized micelles and their use as adjuvants in immunology, J. Pharm. Sci. 65 (1976) 1763-1766.

[11] B. Morein, B. Sundquist, S. Hoglund, K. Dalsgaard, A. Osterhaus, Iscom, a novel struc ture for antigenic presentation of membrane proteins from enveloped viruses, Nature 308 (1984) 457-460.

[12] C. Rueckert, C.A. Guzman, Vaccines: from empirical development to rational design, PLoS Pathog. 8 (2012) e1003001.

[13] J.A. Hubbell, S.N. Thomas, M.A. Swartz, Materials engineering for immunomodulation, Nature 462 (2009) 449-460

[14] K.K. McKinstry, T.M. Strutt, S.L. Swain, Hallmarks of CD4 T cell immunity against influenza, J. Intern. Med. 269 (2011) 507-518.
[15] J.P. Amorij, G.F. Kersten, V. Saluja, W.F. Tonnis, W.L. Hinrichs, B. Slutter, S.M. Bal, J.A. Bouwstra, A. Huckriede, W. Jiskoot, Towards tailored vaccine delivery: needs, challenges and perspectives, J. Control. Release 161 (2012) 363-376.

[16] A.S. De Groot, M. Ardito, F. Terry, L. Levitz, T.M. Ross, L. Moise, W. Martin, Low immunogenicity predicted for emerging avian-origin H7N9: implication for influenza vaccine design, Hum. Vaccin. Immunother. 9 (2013).

[17] Y. He, R. Rappuoli, A.S. De Groot, R.T. Chen, Emerging vaccine informatics, J. Biomed. Biotechnol. 2010 (2010) 218590.

[18] A.S. Fahy, R.H. Clark, E.F. Glyde, G.L. Smith, Vaccinia virus protein C16 acts intracellularly to modulate the host response and promote virulence, J. Gen. Virol. 89 (2008) 2377-2387.

[19] L. Moise, A.H. Gutierrez, C. Bailey-Kellogg, F. Terry, Q. Leng, K.M. Abdel Hady, N.C. Verberkmoes, M.B. Sztein, P.T. Losikoff, W.D. Martin, A.L. Rothman, A.S. De Groot, The two-faced $\mathrm{T}$ cell epitope: examining the host-microbe interface with JanusMatrix, Hum. Vaccin. Immunother. 9 (2013).

[20] B. Guy, The perfect mix: recent progress in adjuvant research, Nat. Rev. Microbiol. 5 (2007) 505-517

[21] B. Slutter, L. Plapied, V. Fievez, M.A. Sande, A. des Rieux, Y.J. Schneider, E. Van Riet, W. Jiskoot, V. Preat, Mechanistic study of the adjuvant effect of biodegradable nanoparticles in mucosal vaccination, J. Control. Release 138 (2009) 113-121.

[22] S.M. Bal, Z. Ding, E. van Riet, W. Jiskoot, J.A. Bouwstra, Advances in transcutaneous vaccine delivery: do all ways lead to Rome? J. Control. Release 148 (2010) 266-282.

[23] Q. Zhu, J. Talton, G. Zhang, T. Cunningham, Z. Wang, R.C. Waters, J. Kirk, B. Eppler, D.M. Klinman, Y. Sui, S. Gagnon, I.M. Belyakov, R.J. Mumper, J.A. Berzofsky, Large intestine-targeted, nanoparticle-releasing oral vaccine to control genitorectal viral infection, Nat. Med. 18 (8) (2012) 1291-1296.

[24] A. Cerutti, The regulation of IgA class switching, Nat. Rev. Immunol. 8 (2008) 421-434.

[25] E. van Riet, A. Ainai, T. Suzuki, H. Hasegawa, Mucosal IgA responses in influenza virus infections; thoughts for vaccine design, Vaccine 30 (2012) 5893-5900.

[26] P. Matzinger, T. Kamala, Tissue-based class control: the other side of tolerance, Nat. Rev. Immunol. 11 (2011) 221-230.

[27] A.E. Gregory, R. Titball, D. Williamson, Vaccine delivery using nanoparticles, Front. Cell. Infect. Microbiol. 3 (2013) 13.

[28] M.F. Bachmann, G.T. Jennings, Vaccine delivery: a matter of size, geometry, kinetics and molecular patterns, Nat. Rev. Immunol. 10 (2010) 787-796.

[29] E. Frohlich, The role of surface charge in cellular uptake and cytotoxicity of medical nanoparticles, Int. J. Nanomedicine 7 (2012) 5577-5591.

[30] J.M. Silva, M. Videira, R. Gaspar, V. Preat, H.F. Florindo, Immune system targeting by biodegradable nanoparticles for cancer vaccines, J. Control. Release 168 (2013) 179-199.

[31] S.Y. Seong, P. Matzinger, Hydrophobicity: an ancient damage-associated molecular pattern that initiates innate immune responses, Nat. Rev. Immunol. 4 (2004) 469-478.

[32] D.T. O'Hagan, G.S. Ott, G.V. Nest, R. Rappuoli, G.D. Giudice, The history of MF59((R)) adjuvant: a phoenix that arose from the ashes, Expert Rev. Vaccines 12 (2013) 13-30.

[33] J.A. Champion, Y.K. Katare, S. Mitragotri, Particle shape: a new design parameter for micro- and nanoscale drug delivery carriers, J. Control. Release 121 (2007) 3-9.

[34] J.A. Champion, S. Mitragotri, Role of target geometry in phagocytosis, Proc. Natl. Acad. Sci. U. S. A. 103 (2006) 4930-4934.

[35] R. Vacha, F.J. Martinez-Veracoechea, D. Frenkel, Receptor-mediated endocytosis of nanoparticles of various shapes, Nano Lett. 11 (2011) 5391-5395.

[36] K. Niikura, T. Matsunaga, T. Suzuki, S. Kobayashi, H. Yamaguchi, Y. Orba, A. Kawaguchi, H. Hasegawa, K. Kajino, T. Ninomiya, K. Ijiro, H. Sawa, Gold nanoparticles as a vaccine platform: influence of size and shape on immunological responses in vitro and in vivo, ACS Nano 7 (5) (2013) 3926-3938.

[37] A. Jegerlehner, T. Storni, G. Lipowsky, M. Schmid, P. Pumpens, M.F. Bachmann, Regulation of $\operatorname{IgG}$ antibody responses by epitope density and CD21-mediated costimulation, Eur. J. Immunol. 32 (2002) 3305-3314

[38] B. Bottazzi, A. Doni, C. Garlanda, A. Mantovani, An integrated view of humoral innate immunity: pentraxins as a paradigm, Annu. Rev. Immunol. 28 (2010) 157-183.

[39] M. Kanekiyo, C.J. Wei, H.M. Yassine, P.M. McTamney, J.C. Boyington, J.R. Whittle, S.S. Rao, W.P. Kong, L. Wang, G.J. Nabel, Self-assembling influenza nanoparticle vaccines elicit broadly neutralizing H1N1 antibodies, Nature 499 (7456) (2013) 102-106.

[40] C. Savard, A. Guerin, K. Drouin, M. Bolduc, M.E. Laliberte-Gagne, M.C. Dumas, N. Majeau, D. Leclerc, Improvement of the trivalent inactivated flu vaccine using PapMV nanoparticles, PLoS One 6 (2011) e21522.

[41] Y. van Kooyk, W.W. Unger, C.M. Fehres, H. Kalay, J.J. Garcia-Vallejo, Glycan-based DC-SIGN targeting vaccines to enhance antigen cross-presentation, Mol. Immunol. 55 (2) (2013) 143-145.

[42] O. Lunov, T. Syrovets, C. Loos, G.U. Nienhaus, V. Mailander, K. Landfester, M. Rouis, T. Simmet, Amino-functionalized polystyrene nanoparticles activate the NLRP3 inflammasome in human macrophages, ACS Nano 5 (2011) 9648-9657.

[43] K. Shortman, Y.J. Liu, Mouse and human dendritic cell subtypes, Nat. Rev. Immunol. 2 (2002) 151-161.

[44] T. Kaisho, Pathogen sensors and chemokine receptors in dendritic cell subsets, Vaccine 30 (2012) 7652-7657.

[45] E. Klechevsky, J. Banchereau, Human dendritic cells subsets as targets and vectors for therapy, Ann. N. Y. Acad. Sci. 1284 (2013) 24-30.

[46] M.B. Teunissen, M. Haniffa, M.P. Collin, Insight into the immunobiology of human skin and functional specialization of skin dendritic cell subsets to innovate intradermal vaccination design, Curr. Top. Microbiol. Immunol. 351 (2012) 25-76.

[47] S.S. Diebold, Activation of dendritic cells by toll-like receptors and C-type lectins, Handb. Exp. Pharmacol. (2009) 3-30.

[48] G. Ahlen, L. Strindelius, T. Johansson, A. Nilsson, N. Chatzissavidou, M. Sjoblom, U. Rova, J. Holgersson, Mannosylated mucin-type immunoglobulin fusion 
proteins enhance antigen-specific antibody and T lymphocyte responses, PLoS One 7 (2012) e46959.

[49] B.Y. Chua, D. Johnson, A. Tan, L. Earnest-Silveira, T. Sekiya, R. Chin, J. Torresi, D.C. Jackson, Hepatitis C VLPs delivered to dendritic cells by a TLR2 targeting lipopeptide results in enhanced antibody and cell-mediated responses, PLoS One 7 (2012) e47492.

[50] D. Raghuwanshi, V. Mishra, M.R. Suresh, K. Kaur, A simple approach for enhanced immune response using engineered dendritic cell targeted nanoparticles, Vaccine 30 (2012) 7292-7299.

[51] A. Ejaz, C.G. Ammann, R. Werner, G. Huber, V. Oberhauser, S. Horl, S. Schimmer, U. Dittmer, D. von Laer, H. Stoiber, Z. Banki, Targeting viral antigens to CD11C on dendritic cells induces retrovirus-specific T cell responses, PLoS One 7 (2012) e45102.

[52] L.J. Cruz, P.J. Tacken, J.M. Pots, R. Torensma, S.I. Buschow, C.G. Figdor, Comparison of antibodies and carbohydrates to target vaccines to human dendritic cells via DC-SIGN, Biomaterials 33 (2012) 4229-4239.

[53] P.J. Tacken, I.S. Zeelenberg, L.J. Cruz, M.A. van Hout-Kuijer, G. van de Glind, R.G. Fokkink, A.J. Lambeck, C.G. Figdor, Targeted delivery of TLR ligands to human and mouse dendritic cells strongly enhances adjuvanticity, Blood 118 (2011) 6836-6844.

[54] A.T. Kamath, B. Mastelic, D. Christensen, A.F. Rochat, E.M. Agger, D.D. Pinschewer, P. Andersen, P.H. Lambert, C.A. Siegrist, Synchronization of dendritic cell activation and antigen exposure is required for the induction of Th1/Th17 responses, J. Immunol. 188 (2012) 4828-4837.

[55] T. Kawai, S. Akira, The role of pattern-recognition receptors in innate immunity: update on Toll-like receptors, Nat. Immunol. 11 (2010) 373-384.

[56] M. Yoneyama, K. Onomoto, T. Fujita, Cytoplasmic recognition of RNA, Adv. Drug Deliv. Rev. 60 (2008) 841-846.

[57] K. Schroder, J. Tschopp, The inflammasomes, Cell 140 (2010) 821-832.

[58] J.M. Blander, R. Medzhitov, Toll-dependent selection of microbial antigens for presentation by dendritic cells, Nature 440 (2006) 808-812.

[59] S. Burgdorf, A. Kautz, V. Bohnert, P.A. Knolle, C. Kurts, Distinct pathways of antigen uptake and intracellular routing in CD4 and CD8 T cell activation, Science 316 (2007) 612-616.

[60] R. Belizaire, E.R. Unanue, Targeting proteins to distinct subcellular compartments reveals unique requirements for MHC class I and II presentation, Proc. Natl. Acad. Sci. U. S. A. 106 (2009) 17463-17468.

[61] L. Cervi, A.S. MacDonald, C. Kane, F. Dzierszinski, E.J. Pearce, Cutting edge: dendritic cells copulsed with microbial and helminth antigens undergo modified maturation, segregate the antigens to distinct intracellular compartments, and concurrently induce microbe-specific Th1 and helminth-specific Th2 responses, J. Immunol. 172 (2004) 2016-2020.

[62] C. Guiducci, G. Ott, J.H. Chan, E. Damon, C. Calacsan, T. Matray, K.D. Lee, R.L. Coffman, F.J. Barrat, Properties regulating the nature of the plasmacytoid dendritic cell response to Toll-like receptor 9 activation, J. Exp. Med. 203 (2006) 1999-2008.

[63] C. Keijzer, B. Slutter, R. van der Zee, W. Jiskoot, W. van Eden, F. Broere, PLGA, PLGATMC and TMC-TPP nanoparticles differentially modulate the outcome of nasal vaccination by inducing tolerance or enhancing humoral immunity, PLoS One 6 (2011) e26684

[64] J. Kunisawa, S. Nakagawa, T. Mayumi, Pharmacotherapy by intracellular delivery of drugs using fusogenic liposomes: application to vaccine development, Adv. Drug Deliv. Rev. 52 (2001) 177-186.

[65] N. Budimir, T. Meijerhof, J. Wilschut, A. Huckriede, A. de Haan, The role of membrane fusion activity of a whole inactivated influenza virus vaccine in (re)activation of influenza-specific cytotoxic T lymphocytes, Vaccine 28 (2010) 8280-8287.

[66] G. Napolitani, A. Rinaldi, F. Bertoni, F. Sallusto, A. Lanzavecchia, Selected Toll-like receptor agonist combinations synergistically trigger a T helper type 1-polarizing program in dendritic cells, Nat. Immunol. 6 (2005) 769-776.

[67] P. Johansen, D. Mohanan, J.M. Martinez-Gomez, T.M. Kundig, B. Gander, Lymphogeographical concepts in vaccine delivery, J. Control. Release 148 (2010) 56-62.

[68] P. Johansen, Y. Men, R. Audran, G. Corradin, H.P. Merkle, B. Gander, Improving stability and release kinetics of microencapsulated tetanus toxoid by co-encapsulation of additives, Pharm. Res. 15 (1998) 1103-1110.

[69] G. Boehm, M. Peyre, D. Sesardic, R.J. Huskisson, F. Mawas, A. Douglas, D. Xing, H.P. Merkle, B. Gander, P. Johansen, On technological and immunological benefits of multivalent single-injection microsphere vaccines, Pharm. Res. 19 (2002) 1330-1336.

[70] S.A. Plotkin, Complex correlates of protection after vaccination, Clin. Infect. Dis. 56 (2013) 1458-1465.

[71] M. Srinivas, L.J. Cruz, F. Bonetto, A. Heerschap, C.G. Figdor, I.J. de Vries, Customizable, multi-functional fluorocarbon nanoparticles for quantitative in vivo imaging using 19F MRI and optical imaging, Biomaterials 31 (2010) 7070-7077.

[72] C. Primard, N. Rochereau, E. Luciani, C. Genin, T. Delair, S. Paul, B. Verrier, Traffic of poly(lactic acid) nanoparticulate vaccine vehicle from intestinal mucus to subepithelial immune competent cells, Biomaterials 31 (2010) 6060-6068.

[73] N. Desai, Challenges in development of nanoparticle-based therapeutics, AAPS J. 14 (2012) 282-295.

[74] S.E. McNeil, Nanoparticle therapeutics: a personal perspective, Wiley Interdiscip. Rev. Nanomed. Nanobiotechnol. 1 (2009) 264-271.

[75] L. Yildirimer, N.T. Thanh, M. Loizidou, A.M. Seifalian, Toxicology and clinical potential of nanoparticles, Nano Today 6 (2011) 585-607.

[76] G. Smith, R. Raghunandan, Y. Wu, Y. Liu, M. Massare, M. Nathan, B. Zhou, H. Lu, S. Boddapati, J. Li, D. Flyer, G. Glenn, Respiratory syncytial virus fusion glycoprotein expressed in insect cells form protein nanoparticles that induce protective immunity in cotton rats, PLoS One 7 (2012) e50852.

[77] G.M. Glenn, G. Smith, L. Fries, R. Raghunandan, H. Lu, B. Zhou, D.N. Thomas, S.P. Hickman, E. Kpamegan, S. Boddapati, P.A. Piedra, Safety and immunogenicity of a Sf9 insect cell-derived respiratory syncytial virus fusion protein nanoparticle vaccine, Vaccine 31 (2013) 524-532.

[78] V. Gerdts, G. Mutwiri, J. Richards, S.V. Hurk, A.A. Potter, Carrier molecules for use in veterinary vaccines, Vaccine 31 (4) (2013) 596-602. 